\title{
Magneto-aerostatic bearing for miniature air turbine
}

\author{
KuAng-Yuh Huang And Keng-Ning Chang ${ }^{\mathrm{a}}$ \\ Department of Mechanical Engineering, No. 1, Sec. 4, Roosevelt Road, 10617 Taipei, Taiwan (R.O.C.)
}

Received 3 April 2014, Accepted 7 July 2014

\begin{abstract}
Under the circumstance of pursuing high speed and miniaturization, mini-type air turbines have been widely applied to high-speed dental handpieces in recent years. Among all the components of a mini-type air turbine, bearing is the most important part which significantly affects its efficiency. Friction, collision, and wear are the main causes to make the ball bearing unable to reach higher efficiency. Though very small sliding friction can be realized by the aerostatic bearing, its poor bearing capacity limits the application. This research combines the magnetic and aerostatic levitation principle to develop a magnetoaerostatic hybrid bearing for miniature air turbine. The aerostatic bearing undertakes the main function of the radial and the axial bearings. However, the air bearing also possesses a drawback, which is pneumatic hammer effect induced by the air compressibility. In order to eliminate the pneumatic instability, an axial passive magnetic bearing is integrated into the aerostatic bearing. With the magnetic bearing, not only the bearing capacity can be improved, but also the pneumatic hammer effect can be significantly damped. Both theoretical calculation and finite element method (FEM) are used to study the cause and elimination solution of the pneumatic instability. Through experimental testing, the performance of our developed magneto-aerostatic bearing is also verified. The magneto-aerostatic bearing suppresses the axial vibration over $57 \%$, and also enhances its axial bearing capacity by $50 \%$.
\end{abstract}

Key words: Pneumatic hammer effect / flow-induced vibration / air turbine / magneto-aerostatic bearing

\section{Introduction}

As technology and civil industry progress constantly, the quality of oral medicine has attracted more and more attention. High speed dental handpieces have already been essential tools during dental operation. Through the turbine cartridge, the pneumatic energy is converted to rotational kinetic energy, which is used to cut. Besides the dental application, miniature turbine is also utilized in diverse industrial applications by virtue of its high speed performance. For convenient maintenance, the dental turbine cartridge is designed to be interchangeable, and its turbine spindle is supported by a set of bearings with axial and radial function. To have more applications, efficiency, and precision machining, miniature air turbine must provide steadier and higher speed to generate enough power to cut. Present dental turbine cartridge utilizes a set of ball bearings to support the high speed spindle. However, due to the contact friction, vibration and noise are induced unavoidably. These negative phenomena not only affect the auditory sense and comfort of the patient but also diminish its efficiency and longevity.

\footnotetext{
${ }^{a}$ Corresponding author: b93501075@ntu.edu.tw
}

In order to enhance durability and decrease noise and vibration in high speed operation, non-contact bearings ought to be utilized in miniature dental turbine cartridge. There are several forms of non-contact bearings which are developed and used in different fields, including magnetic, pneumatic, electric, and fluidic principles. In consideration of safety and size limitation, only air and magnetic bearings can be integrated into the miniature dental turbine cartridge to levitate the spindle. Air bearings use the pressure or flowing energy of air film to create the bearing force. Because of its low viscosity, the friction between rotor and stator can be minimized. In comparison with the contact bearings, the air bearings can significantly reduce frictional loss and enhance energy utilization. Besides, various thermal influences produced by frictional loss can also be effectively under control.

Another characteristic of air bearings is that the selfhomogenization effect of the pressurized air can also compensate for some machining errors on the bearing surface. Yoshimoto [1] utilized the Reynolds equation to acquire the dimensions of disparate aerostatic bearings. Sugai and Nakayama [2] proposed that the rotation speed of the dental air turbine can be increased by $50 \%$ or higher by integrating with aerostatic bearings. Fukuyama [3] utilized the porous material in aerostatic bearings to generate the 
flow-restricting effect. Mizumoto and Matsubara [4] investigated the axial stiffness of axial aerostatic bearings experimentally. Holmes and Sykes [5] researched the vibration suppression effect of double-row radial aerostatic bearings. Togo [6] summarized the skill of design and manufacture of air bearings, including porous, aerostatic, and aerodynamic bearings. Matta et al. [7] developed an experimental work of measuring the rotor dynamic coefficients of air bearing. The experimental coefficients are also compared with theoretical results.

The size of the air turbine cartridge utilized in dental handpieces is limited by its dimensions of outside diameter $10 \mathrm{~mm} \times$ length $12 \mathrm{~mm}$. Besides the spindle, turbine rotor, and housing, the bearing can only occupy $1 / 3$ total volume of the air turbine cartridge. Because the bearing capacity is closely related to the bearing surface area, the constrained size of the air turbine cartridge brings out a large challenge to achieve sufficient bearing capacity. Furthermore, pressurized air used in aerostatic bearings may also induce instability because of air compressibility. Hence, we come out to an idea to combine a magnetic levitation with an aerostatic bearing to form a hybrid bearing. The magnetic levitation affects the aerostatic bearing by its magnetic attractive or repulsive force to improve stability and performance. For achieving magnetic levitation, there are mainly two kinds of approaches, passive magnet and active electromagnetic coil. Because of the increment of energy density of permanent magnet, the permanent magnet becomes more and more suitable in various industrial applications. Early researches about passive magnetic levitation focus on deriving the formula of force and stiffness in different aliments of permanent magnets. Bakers [8] researched the direction of alternating magnetization, and proposed an optimal parameter, and it was confirmed by experiment. After that, Groom and Antaki [9] extended its application, and researched the stiffness and loading capacity in stacked-structure radial bearing. For achieving a requiring loading capacity under minimum magnet volume, Yoo et al. [10] investigated two types of magnet aliment for thrust bearing, and compared their performance theoretically and experimentally. Sobotka and Lange [11] researched different configurations of passive magnetic bearings. After that, Biedinger and Lemoine [12] theoretically studied the relation between magnetic performance and dimensional parameters of permanent magnet with various shapes. Jones [13] analyzed the characteristics and reliability of a magnetic bearing. As for the hybrid bearing idea, because of the non-contact characteristic of air and magnetic bearing, several investigations have been implemented to combine their advantages. Morosi and Santo proposed a combination of gas and magnetic levitation technology to improve bearing characteristic [14]. Moreover, a multiphysics model, including solid mechanics, fluid dynamic, and magnetic field is also built theoretically and verified experimentally [15]. The hybrid bearing is also applied to precision moving table. A linear stage [16] with air bearing was designed, and it was preloaded magnetically to eliminate the motion errors. Tan and Li [17] applied

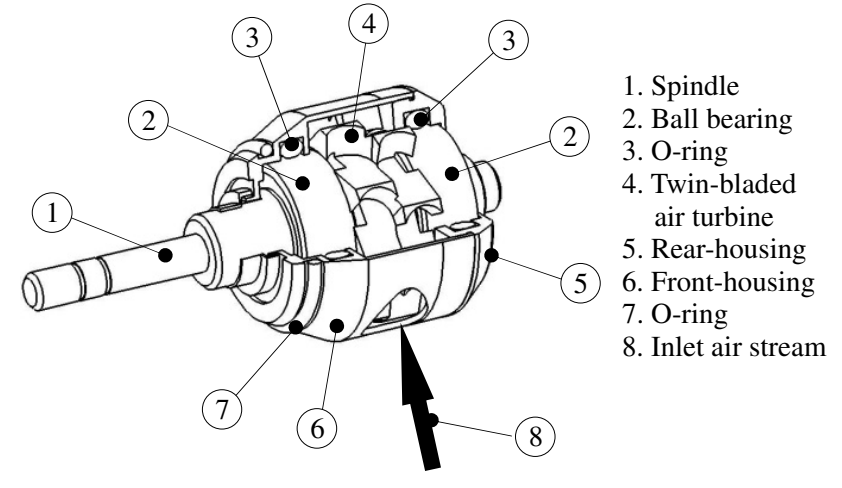

Fig. 1. Conventional turbine cartridge with ball bearing.

permanent magnet to create repulsive force to the hydrodynamic bearing. Chen [18] combined magnetic and aerostatic bearings to achieve high speed, high loading, and high stability. Although passive permanent magnets have lower degree of robustness compared with active magnetic bearing, active electromagnetic coil with electric leakage concern is not allowed to be applied in the dental operation circumstance. Therefore, only permanent magnet can be adopted in this tiny hybrid bearing.

\section{Design and development}

\subsection{Concept}

Figure 1 shows the construction of the mini type air turbine cartridge, which is composed of an air turbine, a spindle, and two deep-groove radial ball bearings in confined housing. The air turbine is responsible for the energy transformation from pneumatic energy to mechanical rotation energy, and it can rotate at a maximum speed of $300 \mathrm{krpm}$. Under a high-speed operation, noise and wear will be intensively generated by friction contact between roller and ring in ball bearing. Furthermore, the friction induces thermal losses and also interferes the stability and efficiency of the spindle. Though the ceramic bearing is used to improve its wear resistance, friction induced collisional vibration is still a disturbance. To improve its durability and lifetime, a non-contact type bearing should be an alternative solution. There are several well-known methods to achieve stable and frictionless operation, such as air, fluid, magnetic, and electric bearings. Because of the operation condition of the mini type turbine cartridge, only the aerostatic and magnetic bearing can be applied to realize the non-contact bearing. Moreover, the aerostatic bearing can share the same air source with the air turbine without any extra power source.

The aerostatic bearing generates the bearing force via the pressurized air inside the air gap. A set of orifices and smooth bearing surface builds up the air gap to withstand the bearing load, and the air pressure can be regulated to adapt to different operation conditions. But due to air compressibility, the pneumatic hammer effect will be easily induced by air pockets. Figure 2 shows the schematic 

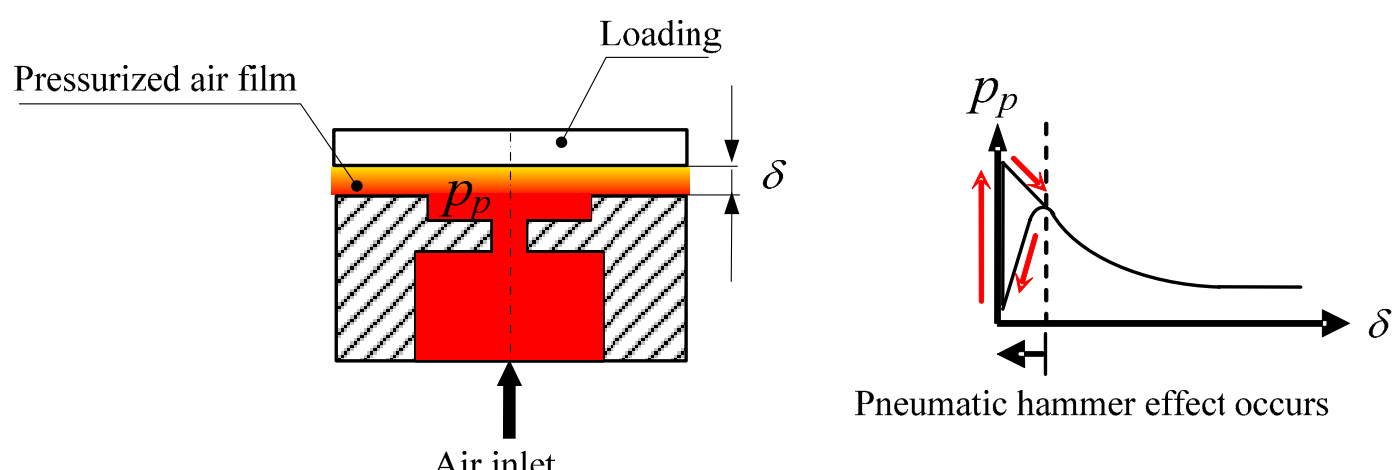

Pneumatic hammer effect occurs

Fig. 2. Pneumatic hammer effect.

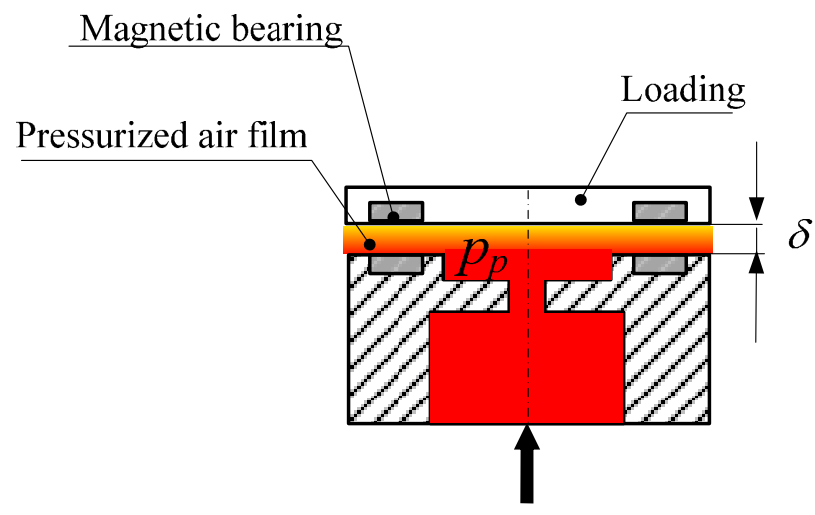

Air inlet

Fig. 3. Combination of magnetic levitation and aerostatic bearing.

of pneumatic hammer effect. For a lessening air gap, the air inside the air pocket is difficult to exhaust due to the fluid viscosity. The pocket pressure $p_{p}$ rapidly accumulates up to $p_{p}+\Delta p$ in a short time, and this pressure increment pushes the bearing surface to a new equilibrium position. For the rapidly expanding air gap, the air exhausts promptly. Once the pressure of the air pocket decreases, the bearing load presses the air gap together so that the pressured air is blocked to build up the pneumatic potential energy.

This periodic variation between pressure and air gap causes the oscillating motion of the bearing surface. Licht et al. [19] proposed stable criteria theorem for single concentric circle thrust air bearing with air pocket. Pan [20] investigated the stability of air bearing through spectral analysis. The pneumatic hammer phenomenon always induces not only vibration but also noise. The operation performance of aerostatic bearing is crucially influenced by the pneumatic hammer effect. In order to reduce the pneumatic hammer effect, the permanent magnets are chosen to integrate into the aerostatic bearing to create magnetic repulsive force to withstand or damp the rapid pneumatic variation, as shown in Figure 3. Each passive magnetic bearing is composed of a set of permanent magnets with repelling arrangement, and its resultant magnetic force can avoid the collision between bearing surfaces. Therefore, the magnetic repelling bearing can increase the bearing capacity and stiffness. By using the strong NdFeB magnet, a high bearing capacity can be easily accomplished in the confined space inside the turbine cartridge.

\subsection{Function and construction}

Figure 4 illustrates the newly-developed air turbine cartridge with magneto-aerostatic hybrid bearing. Two symmetrically installed hybrid bearings replaced the original ceramic-ball-bearing of MicroP company commercial product CHQD-5022 turbine cartridge. Each hybrid bearing is composed of an axial magnetic bearing and a radialaxial aerostatic bearing. A pair of magnetic bearing rotors is attached on both side of the turbine spindle along axial direction. Two bearing stators are fixed firmly to the housing by O-ring to accomplish air-proof and selfaliment function.

Furthermore, the symmetric form of turbine blades mainly induces tangential force to rotate the spindle and less axial force. The aerostatic bearing and the turbine have separated air supply channel. The pressurized air first enters into the air cavity with pressure-stabilizing function, and the air path is divided into radial and axial 


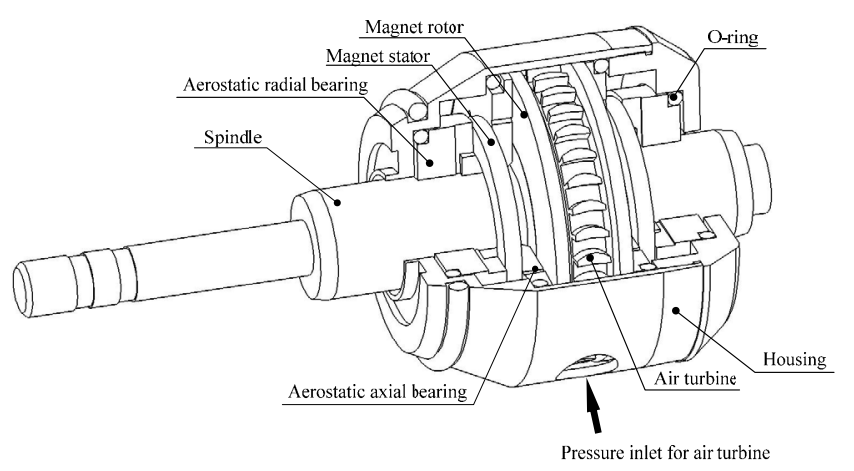

Fig. 4. Magneto-aerostatic bearing integrated into micro turbine cartridge.

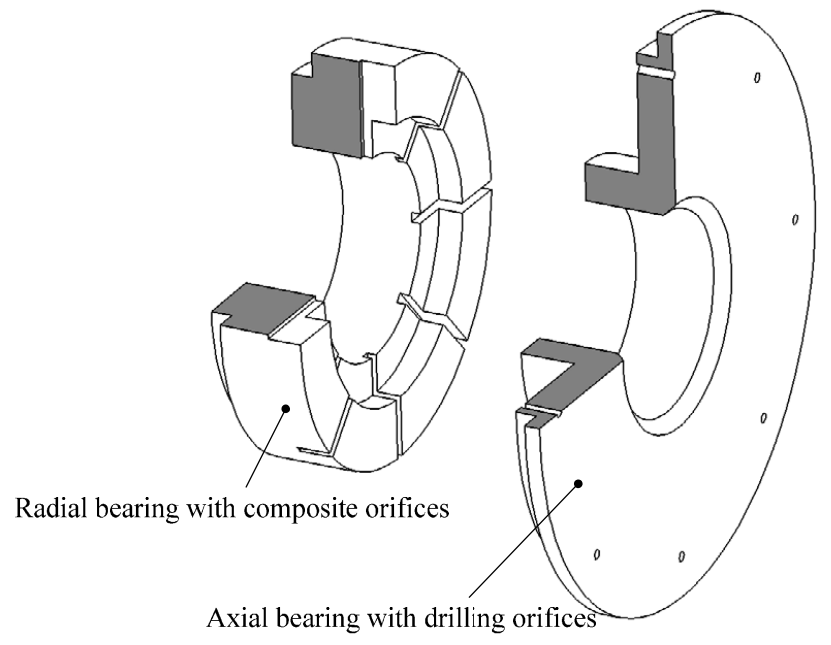

Fig. 5. Composite and drilling orifices for radial and axial aerostatic bearings.

orifices respectively. Based on aerostatic principle, each orifice transforms pressurized air into air film to achieve air levitation. Due to the restricted space inside the turbine cartridge, the fabrication difficulty of the parts is very high, especially those parts for aerostatic bearing. In order to realize the air supply channel and tiny orifices economically, a composite orifice structure of aerostatic bearing is proposed, and is shown in Figure 5. The composite orifices in the aerostatic radial bearing are built up between two parts, only one part is manufactured with orifice groove by using a precision saw blade. The orifice with a large depth to width ratio can increase the air film stiffness and bearing capacity.

\subsection{FEA verification for aerostatic bearing}

Because the aerostatic bearing is combined with axial and radial air film within a compact and limited space, its flow field is difficult to be analyzed. To evaluate the complicated flow field, the FLUENT computational fluiddynamics (CFD) software is applied to investigate the bearing capacity and pneumatic hammer instability, and the simulation model is shown in Figure 6. The model assumptions are as follow: (1) spindle is at axis center (2) zero rotating speed (3) steady state calculation (4)no slip condition for calculating efficiency, the simulation model is simplified into $1 / 4$ axial symmetry. Tetrahedral meshes are applied to the model with 2616250 elements. The size of air gap is relatively small compared with overall model. In order to acquire the precise results in these areas, meshes are refined particularly whose element size is $0.008 \mathrm{~mm}$ and element size in other zone is $0.08 \mathrm{~mm}$.

Taking the following assumptions into consideration: (1) ideal air (2) fully developed boundary laminar flow (3) constant temperature, the axial bearing capacity can be derived from the integration of the pressure distribution on the axial bearing surface.

Axial bearing capacity $F_{a}$ :

$$
\begin{aligned}
\frac{F_{a}}{2 \pi R L p_{s}}=\frac{1}{2 \pi}\left[\int_{0}^{1} \int_{0}^{2 \pi} p_{p} \cos \theta \mathrm{d} \theta \mathrm{d} z\right. \\
\\
\left.\quad+\int_{0}^{1} \int_{0}^{2 \pi}\left(\tau_{x} / p_{s}\right) \sin \theta \mathrm{d} \theta \mathrm{d} z\right]
\end{aligned}
$$

$R$ and $L$ denote the radius and length of the air bearing. $\tau_{x}$ is the shear stress. $p_{s}$ is the inlet pressure, and $p_{p}$ is the pressure distribution on the bearing surface. Figure 7 illustrates the relation between axial bearing capacity and depth of air pocket which has axial air gap of $20 \mu \mathrm{m}$, orifice area of $0.04 \mathrm{~mm}^{2}$, and supply air pressure of 1 bar. The set-up of air pocket is to enhance the ability to store pressure for aerostatic bearing, and it also stands for the "spring effect" because of the compressibility of air. Although the bearing capacity increases with the enlargement of the depth of air packet, the chances for the pneumatic hammer phenomenon to occur are also increased. In order to achieve stable operation of aerostatic bearing, an investigation into the pneumatic hammer instability ought to be processed in different designed parameters. According to the theorem derived by Fuller, a pneumatic hammer occurs as the following inequality is satisfied with:

$$
\frac{s}{q}<\frac{\eta-\varphi}{\alpha+\beta}
$$

where

$$
\begin{aligned}
& s=\frac{\partial M_{\text {sys }}}{\partial \delta}, q=\frac{\partial M_{\text {sys }}}{\partial p_{p}}, \eta=\frac{\partial M_{\text {out }}}{\partial \delta}, \varphi=\frac{\partial M_{\text {in }}}{\partial \delta} \\
& \alpha=-\frac{\partial M_{\text {in }}}{\partial p_{p}}, \beta=\frac{\partial M_{\text {out }}}{\partial p_{p}}
\end{aligned}
$$

$M_{\text {in }}$ and $M_{\text {out }}$ are the mass flow rate for inlet and outlet respectively. $M_{\text {sys }}$ is the gas mass stored in the air gap and pocket. It can be inferred that $s$ is the mass variation which is caused by the air gap $\delta$, i.e. squeeze effect. The $q$ value is the mass variation owing to the pressurized air pocket, i.e. compression effect. For incompressible fluid, the $q$ value equals to zero. Accordingly, it is impossible for the pneumatic hammer effect to occur. In terms of compressible air, the pneumatic hammer effect can be 

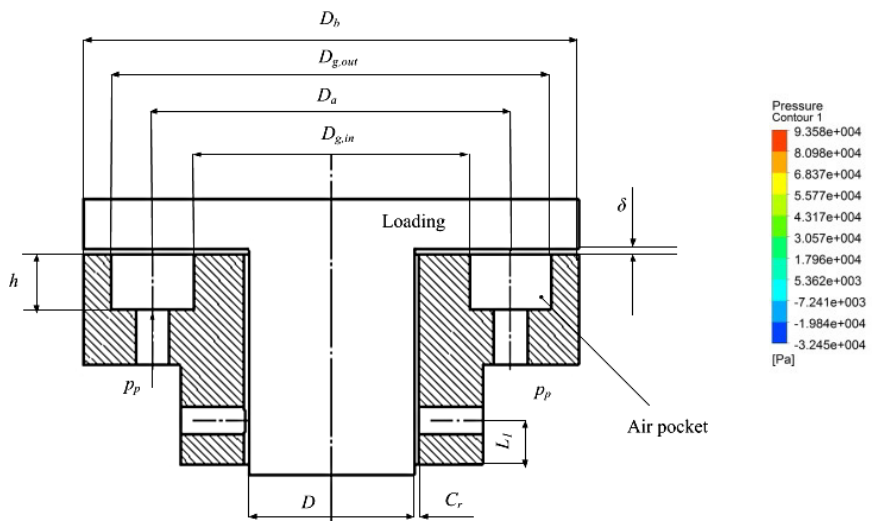

Fig. 6. Simulation model and pressure distribution of aerostatic bearing.

promoted by increasing the supply air pressure, enlarging the volume of air pocket, and reducing an air gap. An axial pneumatic hammer coefficient $P H_{a}$ can be derived by rearranging Equation (2).

$$
P H_{a}=\left(\frac{\eta_{a}-\varphi_{a}}{\alpha_{a}+\beta_{a}}\right) /\left(\frac{s_{a}}{q_{a}}\right)
$$

where

$$
\begin{aligned}
\alpha_{a} & =M_{\text {in }} \xi / p_{p}, \quad \beta_{a}=2 M_{\text {out }} p_{p} /\left(p_{p}^{2}-p_{a}^{2}\right), \\
\eta_{a} & =3 M_{\text {out }} / \delta, \quad \varphi_{a}=M_{\text {in }} / \delta+h \\
q_{a} & =\left\{A_{e}+\frac{1}{4} \pi\left(D_{g, \text { out }}^{2}-D_{g, \text { in }}^{2}\right) h\right\} / R_{0} T, \\
s_{a} & =\left\{A_{e}\left(p_{a}-p_{a}\right)+A p_{a}\right\} / R_{0} T
\end{aligned}
$$

And radial pneumatic hammer coefficient is:

$$
P H_{r}=\left(\frac{\eta_{r}-\varphi_{r}}{\alpha_{r}+\beta_{r}}\right) /\left(\frac{s_{r}}{q_{r}}\right)
$$

where

$$
\begin{aligned}
\alpha_{r} & =M_{\text {in }} \xi / p_{p}, \beta_{r}=2 M_{\text {out }} p_{p} /\left(p_{p}^{2}-p_{a}^{2}\right), \\
\eta_{r} & =3 M_{\text {out }} / C_{r}, \quad \varphi_{r}=M_{\text {in }} / C_{r} \\
q_{r} & =4 \pi D C_{r} L \frac{p_{p}^{2}+2 p_{p} p_{a}}{3 R_{0} T\left(p_{p}+p_{a}\right)^{2}}, \\
s_{r} & =4 \pi D C_{r} L \frac{p_{p}^{2}+p_{p} p_{a}+p_{a}^{2}}{3 R_{0} T\left(p_{p}+p_{a}\right)}
\end{aligned}
$$

The dimensionless mass flow parameter $\xi$ is concerned with the flow-restricting performance, and it is determined by $p_{p} / p_{s}$. A pneumatic hammer phenomenon takes place when $P H$ coefficient exceeds 1 . For outer-groove diameter $D_{g, \text { out }} 9 \mathrm{~mm}$, inner-groove diameter $D_{g, \text { in }} 4 \mathrm{~mm}$, overall bearing diameter $D_{b} 10 \mathrm{~mm}$, radial air gap $C_{r}$ $0.03 \mathrm{~mm}$, and position of orifice $D_{a} 6.5 \mathrm{~mm}$, Figure 8 illustrates the relation between the axial pneumatic hammer coefficient and air gap in different air pocket depths

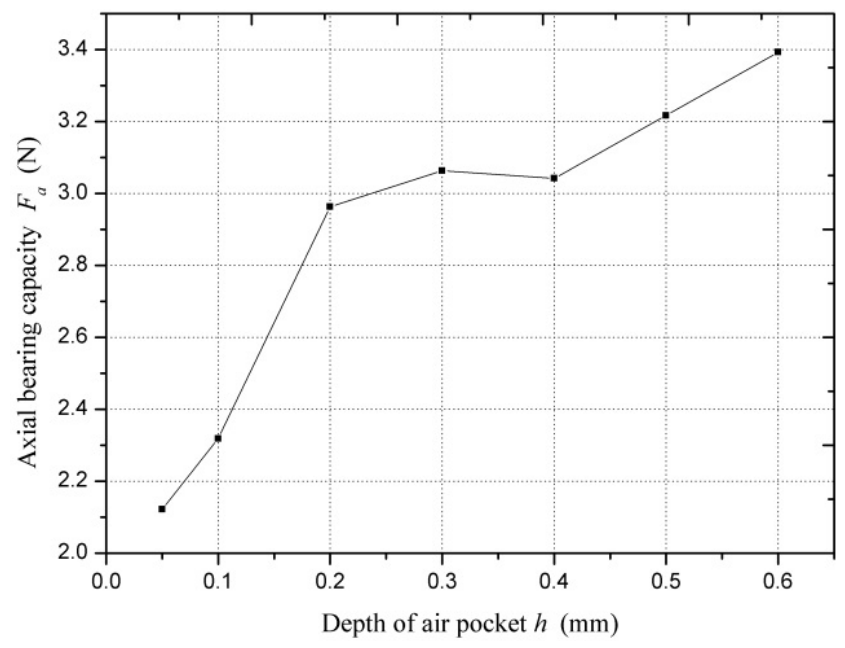

Fig. 7. Relation between axial loading capacity $F_{a}$ and depth of air pocket $h$.

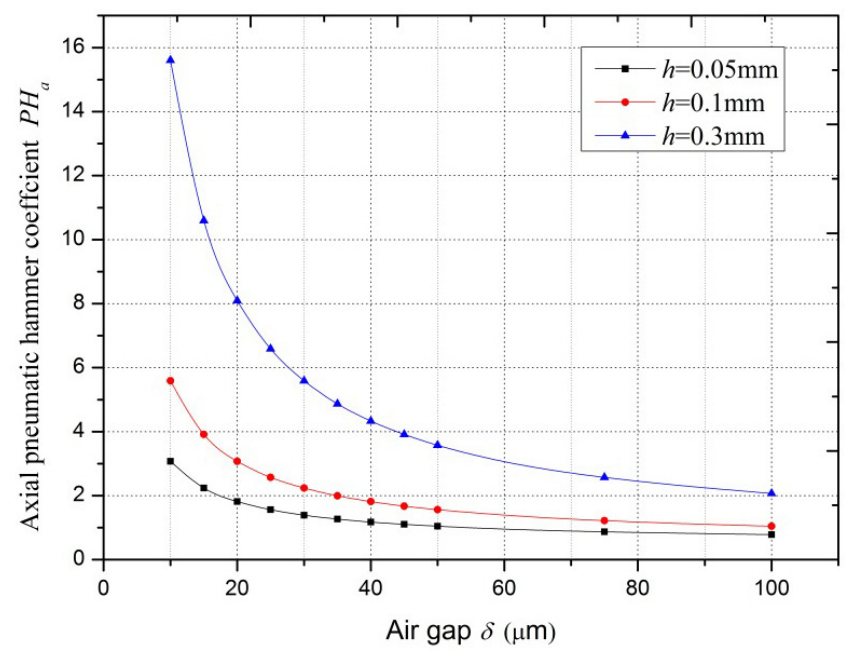

Fig. 8. Axial pneumatic hammer coefficient $P H_{a}$ versus air gap $\delta$ for different depths of air pocket $h$. 


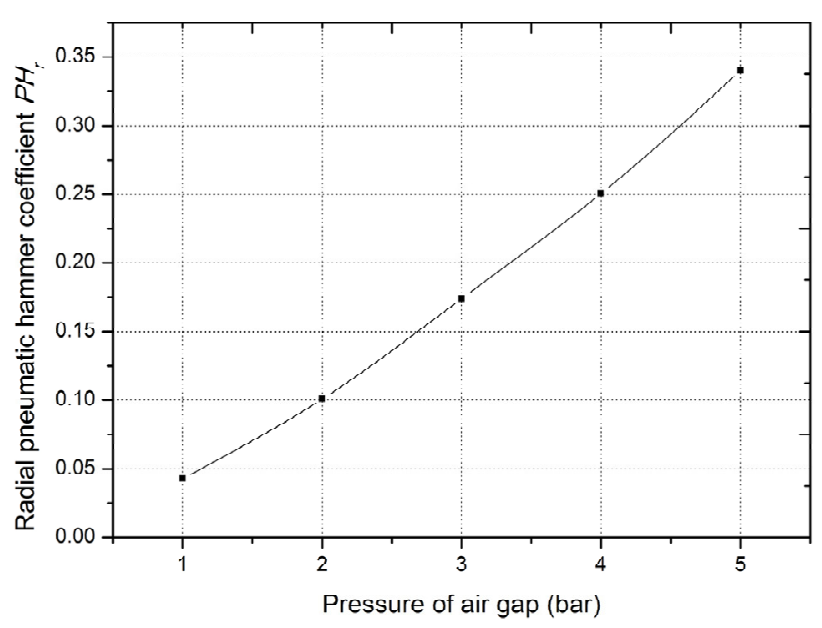

Fig. 9. Radial pneumatic hammer coefficient $P H_{r}$ in different pressures of air gap.

with 4 bar supply air pressure. It can be inferred that a large air pocket and a thin air gap are beneficial to trigger a pneumatic hammer effect.

Figure 9 shows the radial pneumatic hammer coefficient in different pressures of air gap, and the radial pneumatic hammer coefficient is lower than 1 . Moreover, the radial pneumatic hammer phenomenon was not observed during experimental operation. The bearing capacity and stability are important performance for aerostatic bearing, and they are closely related to the air gap and the volume of the air pocket. Although a small air gap, a large air pocket, and large supply pressure increase the bearing capacity, they also increase the possibility of the pneumatic hammer effect.

\subsection{FEA verification for magnetic bearing}

The sintered NdFeB-N52 magnet with characteristics of $B_{r}=14.4 \mathrm{kGs}, H_{c i}=11 \mathrm{kOe},(B H)_{\max }=52$ MGOe, outer diameter. $8 \mathrm{~mm}$, inner diameter $5 \mathrm{~mm}$, thickness $0.8 \mathrm{~mm}$, and $\rho=7.8 \mathrm{~g} \cdot \mathrm{cm}^{-3}$ is utilized in our developed axial passive magnetic bearing. For comprehending magnetic performance, the ANSOFT MAXWELL FEAsoftware is applied to model and simulate the magnetic bearing. The formula of magnetic repulsive force $F_{m}$ can be described as:

$$
F_{m}=-\frac{\mu_{0} \overrightarrow{j_{1}} \overrightarrow{j_{2}}}{4 \pi} \oiint \frac{\vec{r}\left(d s_{1} \cdot d s_{2}\right)}{|\vec{r}|^{3}}
$$

where $\mu_{0}$ is permeability of air, $\vec{r}$ is the location vector between the magnet stator and the magnet rotor, and $\vec{j}$ is the magnet force vector. Figure 10 shows the magnetic field strength $H$ between stator magnet and rotor magnet.

The most concentrated magnetic flux area is located between rotor magnet and stator magnet with loading capacity $1 \mathrm{~N}$ and a stiffness of $7.6 \mathrm{~N} . \mathrm{mm}^{-1}$. When pneumatic hammer instability occurs, there is a relative motion between rotor magnet and hybrid bearing. Therefore,

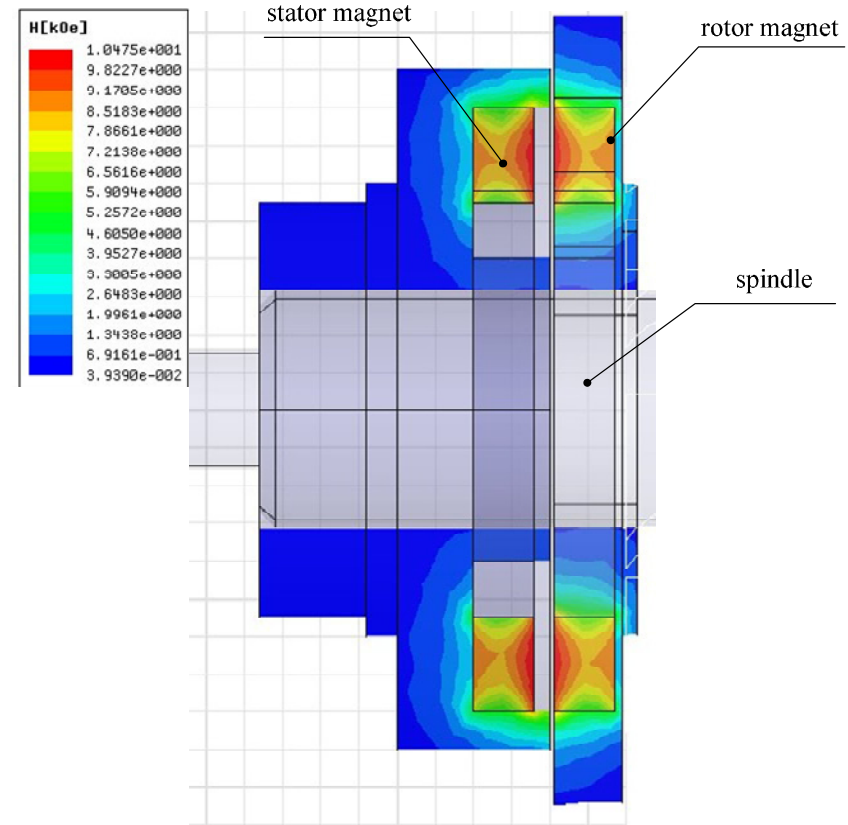

Fig. 10. Magnetic field distribution between stator magnet and rotor magnet.

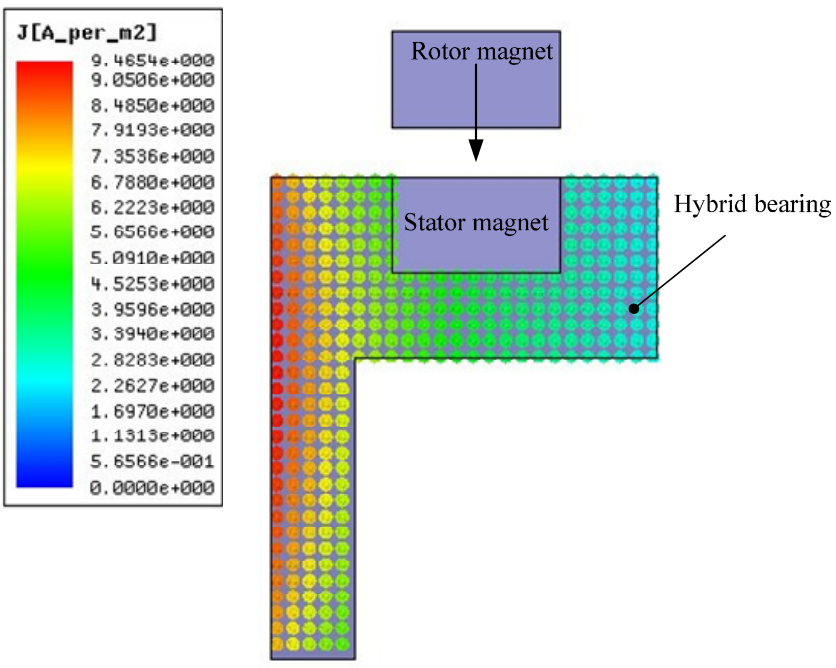

Fig. 11. Eddy current magnitude per area in hybrid bearing.

the eddy current effect should be considered. In order to verify this issue, a simulation about transient response at different time steps is processed. Figure 11 illustrates the eddy current magnitude per area with $2000 \mathrm{~Hz}$ vibration frequency and $0.01 \mathrm{~mm}$ amplitude. Due to the magnetic field variation, the eddy current and inverse magnetic field are also generated to resist magnetic field variation. Through calculating of the electromagnetic field simulation software, about $0.4 \mathrm{~N}$ damping force is induced. 


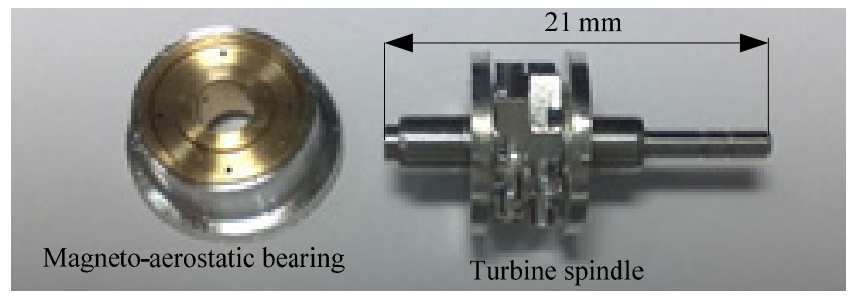

Fig. 12. Realized magneto-aerostatic bearing and turbine spindle.

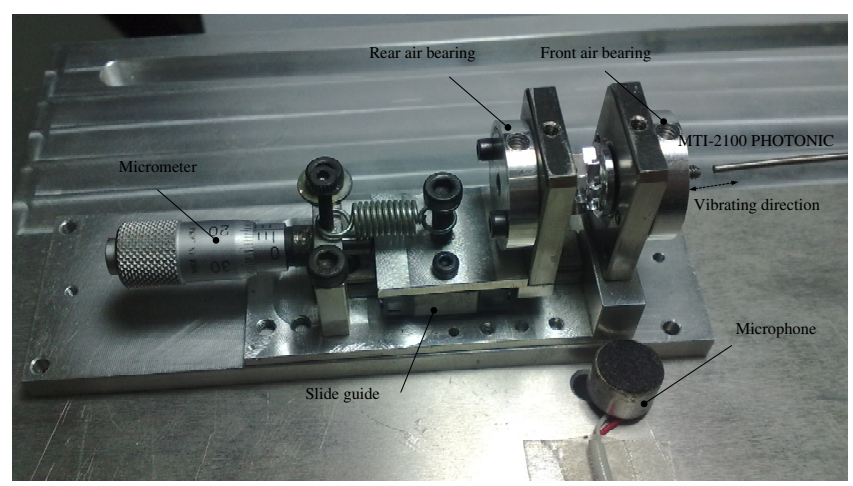

Fig. 13. Experimental set up for running stability.

\section{Experimental verification}

Based on the outcome of FEM result and the construction, the miniature magneto-aerostatic bearing is realized in Figure 12. In order to implement the experimental performance verification, the two magneto-aerostatic bearings are separated into two sets of gap-adjusting structure, whose air gap can be varied through the linear slide guide and micrometer. Before measuring running stability performance, the turbine spindle is processed by the dynamic balancing at G2 level. The non-contact type fiber optic displacement measurement system MTI-2100 PHOTONIC is applied to measure the vibration displacement for the purpose of minimizing the loading effect as much as possible.

\subsection{Running stability and noise}

Figure 13 illustrates the experimental set up of running stability for hybrid bearing. The vibration displacement and noise signal are detected by photonic fiber displacement sensor and microphone respectively, and two of the signals are acquired by NI product MyDAQ simultaneously with a sampling rate $50 \mathrm{k} . \mathrm{s}^{-1}$ and 16 bits resolution. Figure 14 illustrates the relation between vibration frequency and axial air gap in different supply air-pressures and unloading situations. Thanks to the frictionless linear guide of radial aerostatic bearing, the pneumatic hammer phenomenon is triggered without any external disturbance. The vibration frequency is varied depending on the air gap and the supply air pressure.

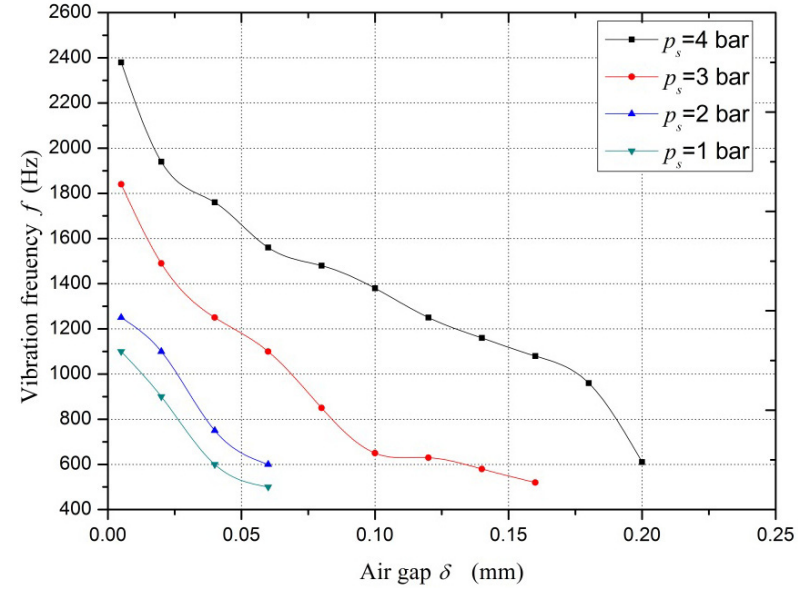

Fig. 14. Influence of air gap $\delta$ on vibration frequency $f$ with different supply pressures $p_{s}$.

It can also be observed that the minimum vibration frequency of the vibrator for occurrence of pneumatic hammer is about $550 \mathrm{~Hz}$.

Figure 15 shows the vibration amplitude and the noise level of the magnetic aerostatic bearing and the aerostatic bearing when the pneumatic hammer phenomenon occurs. The magnetic force makes the aerostatic bearing become a stable equilibrium. The magnetic force increases the equivalent stiffness of hybrid bearing. Moreover, the eddy current effect also generates damping force. Therefore, the repulsive magnetic levitation can effectively suppress and dampen the pneumatic hammer vibration. Furthermore, noise is always produced along with the pneumatic hammer effect, and the hybrid bearing developed in our research can also substantially improve the running stability and reduce noise.

\subsection{Loading test}

The loading experiment setup is presented in Figure 16. The hybrid bearing testing platform is placed against a KYOWA load cell with loading capacity of $20 \mathrm{~N}$. In order to keep a contact relation between the turbine spindle and the load cell, a preload coil spring is applied. The loading signal is connected to a KYOWA commercial signal amplifier DPM-712B, and then it is also acquired by DAQ card. The recovery force which is composed of magnetic and aerostatic force is responsible for bringing the deviated spindle back to its equilibrium position. The relation between axial recovery force and position deviation is shown in Figure 13, whose supply air pressure is 2 bar. It can be inferred that the recovery force of the magneto-aerostatic bearing is 2 times higher than the aerostatic bearing approximately.

\section{Conclusion}

In order to enhance the durability and decrease the noise and vibration, a novel magneto-aerostatic bearing 

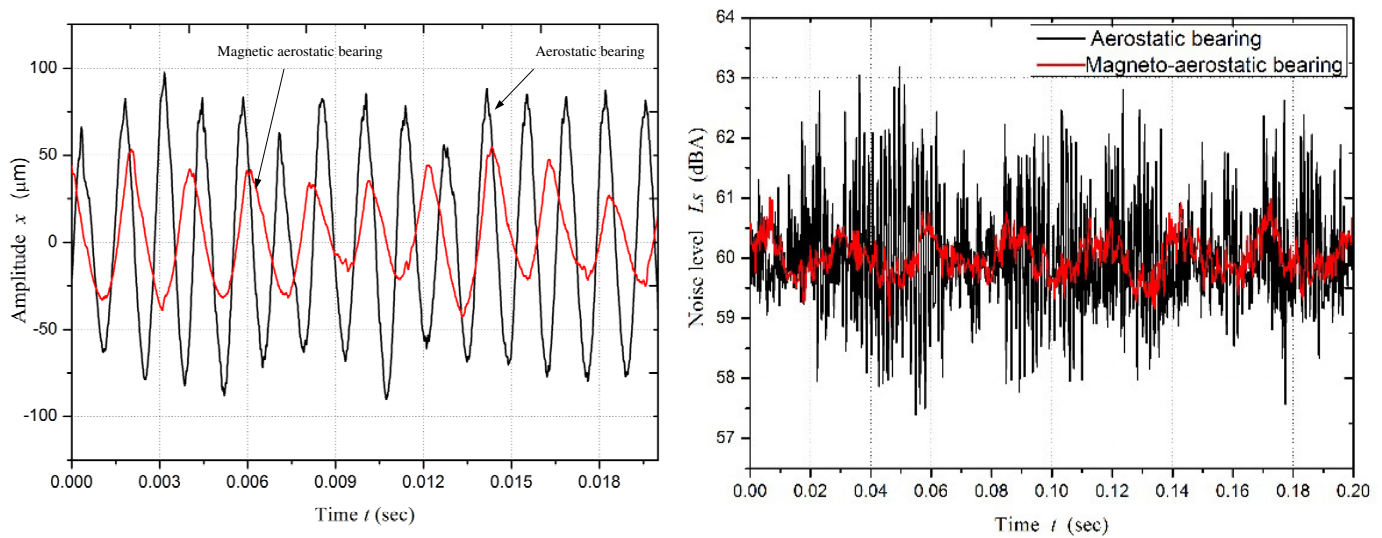

Fig. 15. Axial vibrations and noises induced by aerostatic bearing and magneto-aerostatic bearing.

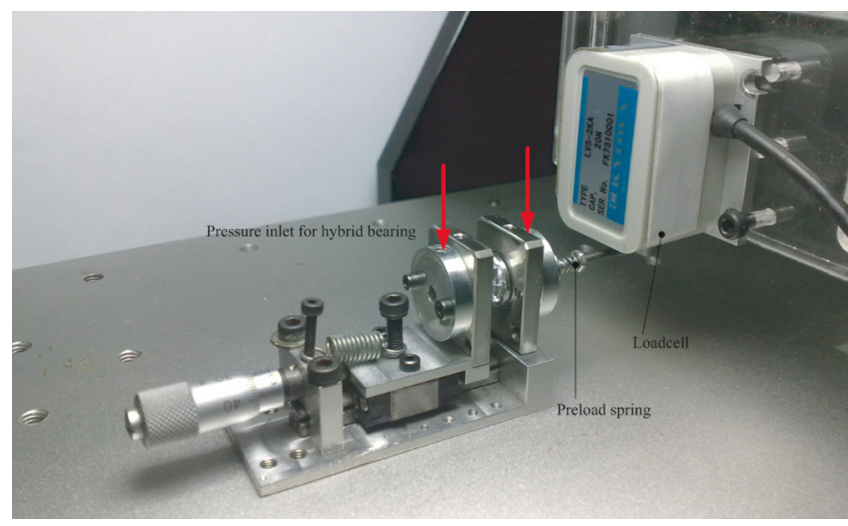

Fig. 16. Experimental setup of loading test.

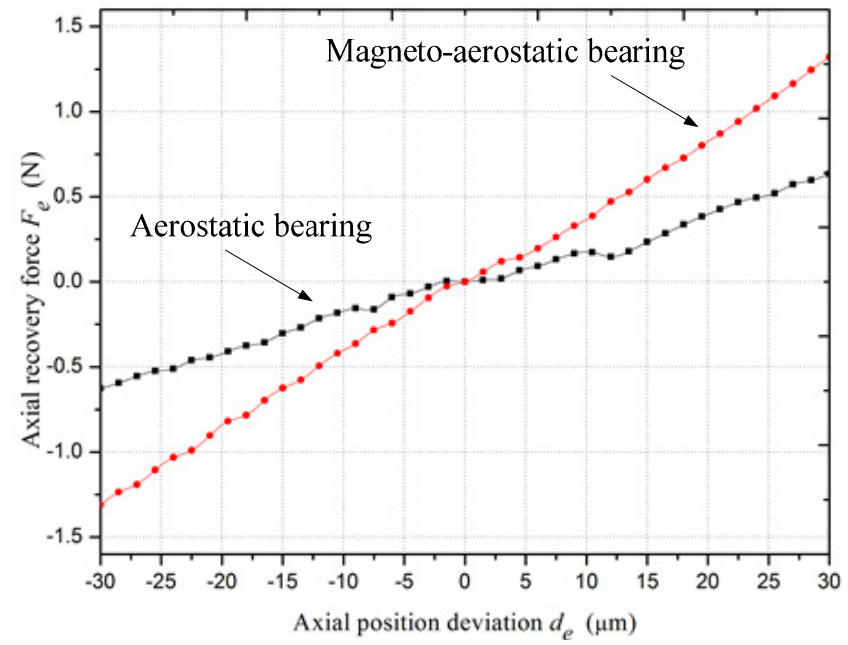

Fig. 17. Axial loading capacities of aerostatic bearing and magneto-aerostatic bearing.

is developed for an air turbine cartridge built in the dental handpiece. By using the same pressurized air source for the air turbine, the aerostatic bearing can provide an axial and radial support for turbine spindle without any friction. However, the aerostatic bearing has a drawback induced by the air compressibility, which is called pneumatic hammer effect. Through unavoidable spindle's run-out induced by external force interference or unbalance, the air gap changes so rapidly that the air film can store potential energy to excite the pneumatic hammer effect. For eliminating the pneumatic hammer effect and enhancing axial loading capacity, a set of $\mathrm{NdFeB}$ passive magnet is integrated into the aerostatic bearing in a very confined space. For realizing precise orifices economically, a composite orifice is proposed. In addition, both theoretical calculation and finite element method (FEM) are used to evaluate the pneumatic hammer effect in this topic. Based on the simulation model and the construction, experiments including the running stability, noise tests, and loading tests are also carried out to verify its performance. The vibration induced by the pneumatic hammer instability is over $57 \%$ improved, and the axial loading capacity is enhanced by $50 \%$.

\section{References}

[1] S. Yoshimoto, Theory and Operating Principle of Aerostatic Bearings, Design Eng. 37 (2002) 51-56

[2] H. Sugai, S. Nakayama, Air bearing for a dental handpiece, U.S. Patent No. 4209293, 1980

[3] H. Fukuyama, Porous, static pressure air bearing device in a dentist's handpiece, U.S. Patent No. 3969822, 1976

[4] H. Mizumoto, T. Matsubara, An aerostatically controlled restrictor for obtaining an infinite stiffness aerostatic thrust bearing, J. Japan Soc. Precision Eng. 55 (1989) $135-140$

[5] R. Holmes, J.E.H. Sykes, The Vibration of an Aeroengine Rotor Incorporating Two Squeeze-film Dampers, J. Aerospace Eng. 210 (1996) 39-51

[6] S. Togo, Air bearing design guide plan, Kyoritsu Publishing, 1985

[7] P. Matta, L. Rudloff, M. Arghir, Experimental investigation of air bearings dynamic coefficients, Mechanics \& Industry 11 (2010) 477-488

[8] F.T. Bakers, A Magnetic Journal Bearing, Philips Tech. Rev. 22 (1961) 232-238

[9] N. Groom, J.F. Antaki, Design Formulas for PermanentMagnet Bearings, Trans. ASME 125 (2003) 734-738 
[10] S.Y. Yoo, W.Y. Kim, S.J. Kim, W.R. Lee, Y.C. Bae, M. Noh, Optimal Design of Non-Contact Thrust Bearing Using Permanent Magnet Rings, Int. J. Precision Eng. Manuf. 12 (2011) 1009-1014

[11] G. Sobotka, R. Lange, Characteristics of a Magnetic Rotor Bearing for Active Vibration Control, Conf. On Vibrations in Rotating Machinery (1976) C239/76

[12] M. Biedinger, D. Lemoine, Shape Sensitivity Analysis of Magnetic Force, IEEE Trans. Magn. 33 (1997) 2309-2316

[13] G. Jones, Magnetic Bearing Can Improve Reliability and Performance of Pumps, World Pumps 366 (1997) 48-49

[14] S. Morosi, I.F. Santos, Stability Analysis of Flexible Rotors Supported by Hybrid Permanent Magnet-Gas Bearings, 11th Pan-American Congress of Applied Mechanics - PACAM XI, 2010, pp. 1-8

[15] S. Morosi, From Hybrid to Actively-Controlled Gas Lubricated Bearings - Theory and Experiment, Ph.D. Thesis, Department of Mechanical Engineering, Technical University of Denmark, 2011
[16] S.K. Ro, S. Kim, Y. Kwak, C.H. Park, A linear air bearing stage with active magnetic preloads for ultraprecise straight motion, Precision Eng. 34 (2010) 186-194

[17] Q.C. Tan, W. Li, B. Liu, Investigations on a permanent magnetic-hydrodynamic hybrid journal bearing, Tribology Int. 35 ( 2002) 443-448

[18] Y.D. Chen, Design and development of hybrid magneticaerostatic bearing and performance investigation, Master thesis of national Taiwan university, 1996

[19] L. Licht, D.D. Fuller, B. Sternlicht, Self-Excited Vibration of an Air Lubricated Thrust Bearing, Trans. ASME 80 (1958) 411-414

[20] C.H.T. Pan, Spectral Analysis of gas bearing system for stability studies, Ninth Midwestern Mechanics Conference, 1965 